\title{
Etude D’un Espace Marchand Specialise Dans La Vente De Medicaments De La Rue: L'exemple Du Marche De Roxy A Adjame (Abidjan, Cote d'Ivoire)
}

\author{
Koffi Bouadi Arnaud Ferrand, (Doctorat De Géographie) \\ Université Felix HOUPHOUET BOIGNY Cocody-Abidjan/Côte d'Ivoire \\ Aloko-N'guessan Jérôme \\ Professeur Titulaire de Géographie à l'Université Felix HOUPHOUET \\ BOIGNY Cocody-Abidjan/Côte d'Ivoire
}

doi: 10.19044/esj.2017.v13n5p388 URL:http://dx.doi.org/10.19044/esj.2017.v13n5p388

\begin{abstract}
This study which has been held on the Roxy market of Adjamé took place from November 2013 to October 2014. It is a transversal study with descriptive and analytical aim whose objective is to proceed to the description of the organization of the circuit of the informal sale of drugs in Abidjan. This article describes the socio-demographic characteristics of street drugs vendors before examining drug pricing methods.

Documentary research, direct observation and interviews with vendors were the main techniques used to gatherg information.

Our investigations show that the sale of drugs at Roxy is done at 95\% by Ivorian women in majority and for the most part illiterate. Pricing is highly dependent on the place of supply of drugs: drugs with a marketing authorization are more expensive on the illicit market than drugs which do not have marketing authorization. In the category of drug products with a marketing authorization: syrups, out-of-stock drugs products in pharmacy and drug the delivery of which asks for time of wait of at least a day in pharmacy is more expensive on the illicit market.
\end{abstract}

Keywords: Street drugs - Roxy market - price - marketing authorization

Resumé

Cette étude sur le marché Roxy d'Adjamé s'est déroulée de novembre 2013 à octobre 2014. C'est une étude transversale à visée descriptive et analytique dont l'objectif est de procéder à la description de l'organisation du circuit de la vente informel de médicaments à Abidjan. Cet article décrit, les caractéristiques sociodémographiques des vendeurs de 
médicaments de la rue, avant de se pencher sur les méthodes de fixation de prix.

La recherche documentaire, l'observation directe et l'exécution d'entretien avec les vendeurs ont été les principales techniques de collecte de l'information.

Il ressort de nos investigations que la vente des médicaments à Roxy mobilise à 95\% des femmes majoritairement de nationalité ivoirienne et pour la plupart analphabètes. La fixation des prix dépend fortement du lieu d'approvisionnement des médicaments: les médicaments disposant d'une autorisation de mise sur le marché sont plus chers sur le marché illicite que les médicaments ne disposant pas d'autorisation de mise sur le marché. Dans la catégorie des médicaments disposant d'une autorisation de mise sur le marché, les sirops, les médicaments en rupture de stock dans les pharmacies et les médicaments dont la délivrance demande un délai d'attente d'au moins un jour dans les pharmacies sont plus chers sur le marché illicite.

Mots clés: Médicaments de la rue - marché Roxy - prix - Autorisation de Mise sur le Marché

\section{Introduction}

La nécessité d'une performance économique et d'un bon fonctionnement des systèmes de santé pour le bien-être individuel passe par un accès à des médicaments de qualité, préalablement sélectionnés puis utilisés de manière rationnelle. Conscients de ce fait, la Côte d'Ivoire s'est dotée dès le lendemain des indépendances d'une politique pharmaceutique devant garantir cette accessibilité en rendant le médicament disponible pour tous. Où que l'on soit sur le territoire, les médicaments devrais être disponible à tout moment dans les structures chargées de sa distribution, à un prix abordable, avec une garantie d'efficacité et de qualité.

Aujourd'hui le marché pharmaceutique ne satisfait que 30\% des besoins de la population ivoirienne (Koulibaly et Toumy, 2014). Cet état des choses a permis la naissance d'un nouveau secteur d'activité : la vente illicite de médicaments de la rue. En l'espace de quelques années seulement, la vente de médicaments de la rue, qui était jadis une pratique isolée, s’exerçant de façon artisanale s`est très vite muée en l’établissement de véritables « industries » qui infectent et encrassent tous les secteurs d'activité. Nous avons assisté à la multiplication de la vente de médicaments de la rue dans toutes les villes de la Cote d'Ivoire et notamment à Abidjan. Il existe à Abidjan un marché qui réunit près de 30 \% des vendeurs de médicaments de la rue de la Cote d'Ivoire (Kouakou, 2007). C'est le marché dénommé Roxy dans la commune d’Adjamé. Il représente le plus grand marché de vente illicite de médicaments. 
Qui sont les animateurs de ce marché ? Quels sont les produits pharmaceutiques vendus sur ce marché ? D’où viennent-ils ? Par quel mécanisme les prix sont-ils fixés sur ce marché ? Autant de questions qui fondent la présente recherche et qui vont structurer notre réflexion.

\section{Méthodologie de collecte et d'approche du terrain}

Notre étude s'est déroulée au marché Roxy situé dans la commune d’Adjamé (figure 1) de novembre 2013 à octobre 2014.

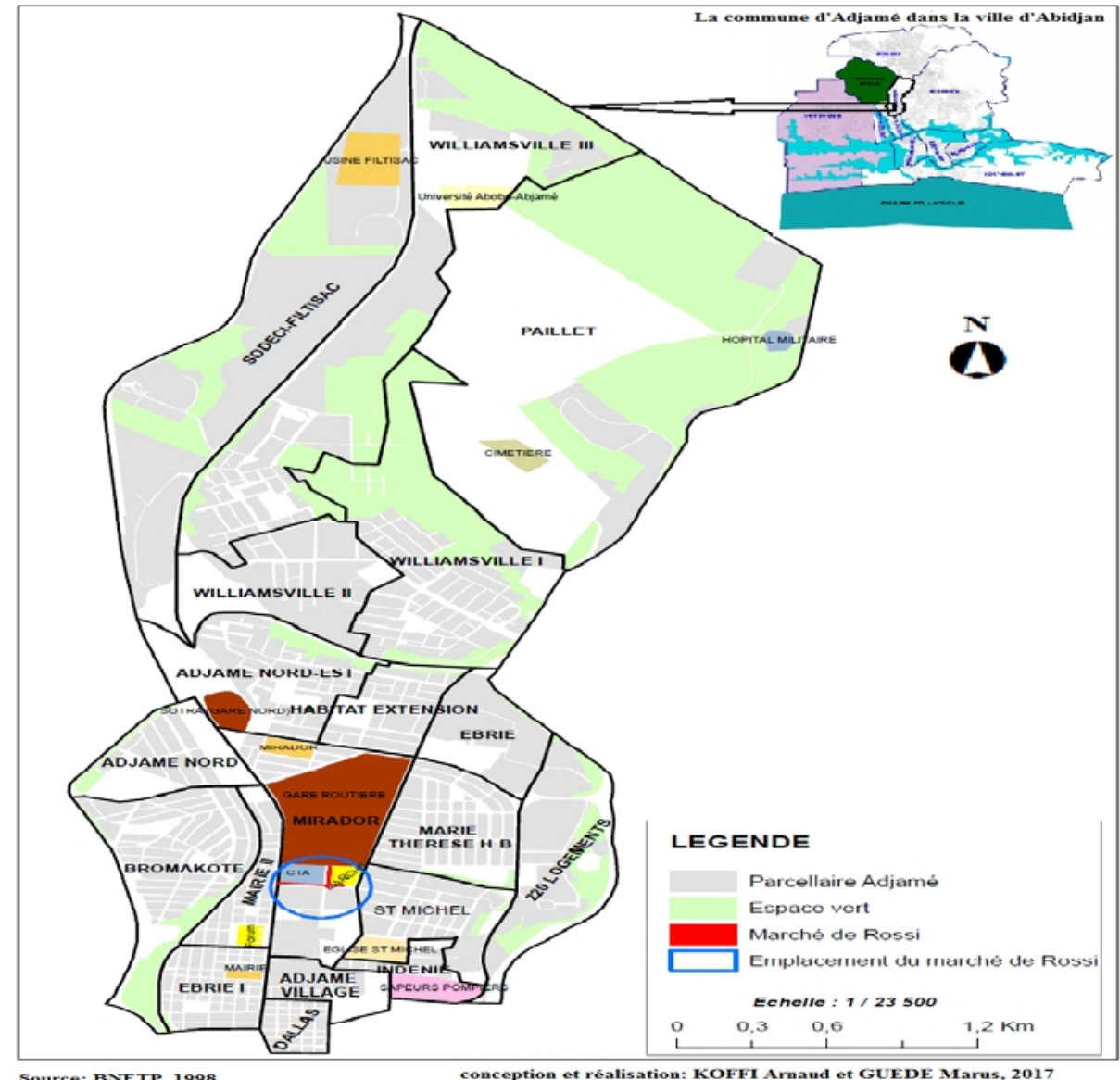

Localisation de la zone d'étude

Cette étude descriptive et analytique a été possible grâce à :

- $\quad$ Des recherches documentaires: nous avons eu recours aux travaux antérieurs afin de nous imprégner de l'état d'avancement scientifique de notre champ d'étude. Cette phase nous a permis d'avoir des connaissances générales sur le sujet par la consultation de thèses, d'articles, d'encyclopédies dans la bibliothèque de l'université de Bordeaux 3 et sur internet. 
- $\quad$ Des entretiens : ils nous ont permis d’identifier les animateurs de ce commerce illicite et de saisir le mécanisme de la fixation des prix des médicaments de la rue à travers un entretien avec des vendeurs. Ne pouvant faire une enquête exhaustive du fait du caractère illicite et du coût qu'elle engendre, nous avons élaboré un échantillonnage. Nous avons opté pour un sondage de 10,40\% compte tenu de l'effectif élevé des acteurs concernés. Pour 2015 vendeurs de médicaments de la rue du marché Roxi à Adjamé (Abidjan) en 2013 (obtenu par un préalable recensement que nous avons effectué), notre calcul nous a amené à sélectionner 210 vendeurs de manière aléatoire. Les 210 vendeurs ont été interrogés à l’intérieur du marché Roxy. La logique est que les vendeurs installés à l’intérieur du marché sont ceux qui peuvent nous aider à une meilleure compréhension du phénomène. Notre guide d'entretien se compose essentiellement de questions non directive afin de laisser les enquêtés donner toutes les informations pouvant nous aider à comprendre leur comportement. Les informations collectées étaient relatives aux sources d'approvisionnements des médicaments de la rue, à des variables sociodémographiques relatives aux vendeurs et aux attitudes relatives aux prix des médicaments.

- Une observation directe : qui nous a permis d'observer le comportement des vendeurs afin de vérifier la véracité de leurs dires, ainsi que les stratégies adoptées par les vendeurs pour conquérir les clients.

\section{Résultat et discussion}

\section{La description du marché Roxy et de l'environnement social de la commercialisation des médicaments de la rue}

Le marché Roxy est situé dans la commune d'Adjamé. Commune située dans le district d'Abidjan, est tournée depuis toujours vers le commerce et le transport. Avec une superficie de 1210 hectares, la commune cosmopolite d'Adjamé compte 19 quartiers avec une population de 372978 habitants selon le Recensement General de la Population et de l'Habitat de 2014. Si longtemps la commune d'Adjamé a toujours été célèbre, c’est de par son marché illicite de médicaments : Roxy.

En effet le marché Roxy tire son nom d'un célèbre cinéma qui a fermé depuis la fin des années 1980. Toutes sortes de médicaments de toutes formes galéniques sont vendues sur ce marché. Vendre des médicaments de la rue sur le marché Roxy passe par un apprentissage. L’apprentissage est un passage obligé pour pratiquer le commerce de médicaments de la rue.

Le nombre de filles qui secondent la vendeuse principale de médicaments de la rue est important, les vendeuses possèdent en moyenne deux filles. Ces filles sont des apprenties. Leurs tâches commencent tôt le matin et consistent à mettre les boîtes vides de médicaments sur les étalages, alors que la vendeuse principale de médicaments de la rue va faire des achats 
ou récupérer les nouveaux arrivages de médicaments et faire d’autres courses avant de venir au marché. 92\% des apprentis ont un lien de parenté avec la vendeuse. Le nombre important des apprentis s'explique par l'abandon volontaire ou forcé de la scolarité. Dans les villages c’est généralement un abandon forcé car les parents n’ont plus les moyens de payer les frais nécessaires à la poursuite de la scolarisation. Les filles sont envoyées en ville, pour aider une tante, une sœur ou un parent éloigné qui est vendeuse de médicaments de la rue. Nombreuses sont les filles qui quittent de leur propre gré leur village pour venir tenter leur chance en ville. Elles proposent aux vendeuses de médicaments de la rue d’être « la fille à tout faire » à leurs domiciles ou sur le lieu de vente.

Ce sont dans certains cas les enfants des vendeuses qui, les weekends et pendant les congés scolaires, viennent aider leur maman sur le marché.

C'est en aidant la vendeuse de médicaments de la rue que les apprentis acquièrent de l'expérience : elles apprennent le nom des médicaments, des maladies qu'ils traitent et surtout la technique de la fixation des prix.

L’apprentissage commencent véritablement avec la vente mobile de médicaments de la même classe thérapeutique (des plaquettes de déparasitant, des seringues, etc.). Au fur et à mesure qu'elles gagnent en expérience, les apprentis ajoutent à leur vente, d'autres classes thérapeutiques, jusqu'à obtenir la maîtrise de la vente de plusieurs classes à la fois. Ainsi une fois le capital de départ constitué avec l'aide des parents, des amis, de la patronne ou du conjoint, l'apprenti devient une vendeuse de médicaments de la rue.

\section{Profil sociodémographique et économique des vendeurs de médicaments de la rue}

Les commerces de vente de médicaments de la rue sont généralement tenus par des personnes qui en sont chef actuellement (95\%). Ces commerces ont, pour les 2/3 d'entre elle une durée de vie supérieur à 5 ans. Elles ne sont enregistrés ni à la mairie, à la patente et encore moins au registre de commerce. Elles ne tiennent pas de comptabilité écrite formelle.

Au sein de la main d'œuvre, les activités de vente de médicaments de la rue sont pratiquées aussi bien par les femmes que par les hommes. Cependant on note une forte présence féminine. Elles représentent $95 \%$ contre $05 \%$ pour les hommes. Nos résultats présentent certaines différences avec les études précédentes, notamment celle réalisé par Abdallah (Abdallah, 2000) qui observe dans son étude une prédominance des vendeurs de sexe masculin. 
En effet, la prédominance des femmes s'explique par le fait que le commerce dans les marchés leur est traditionnellement dévolu. C’est pourquoi, tout au long de notre enquête nous avons remarqué une présence massive de femmes dans l'activité de vente de médicaments de la rue. Les préjugés que les gens ont par rapport à l'activité de vente dans les marchés freinent les hommes dans l'exercice de ces activités (Kouamé, 2007). Le commerce illicite de médicaments est tenu à la fois par les jeunes, les adultes et les personnes âgées. Les jeunes (moins de 18 ans) représentent 06\% des commerçants, les adultes (18 à 55 ans) représentent $87 \%$ et les personnes âgées (55 ans et plus) n'en constituent que $07 \%$. Le pourcentage élevé des adultes est dû au fait que dans cette tranche d'âges les gens sont plus responsables pour mener sa propre activité. La faible représentation des personnes du troisième âge montre qu'ils se consacrent de plus en plus à la formation des jeunes dont ils supervisent parfois la conduite des activités. Par contre, le pourcentage inférieur des jeunes montre que cette tranche d'âge dispose de peu de moyens pour entreprendre. En effet, les jeunes constituent d'ailleurs une population à charge, dépourvue de moyens financiers. Leur présence ici s'explique par le fait que la majorité d'entre eux sont déscolarisés et cherchent tant bien que mal à s’insérer dans la vie active dans le but de subvenir à leur besoins élémentaires : gagner de l'argent pour se nourrir, se soigner et se vêtir. Beaucoup parmi eux mènent ces activités pour le compte de leurs parents. Depuis quelques années, on observe une arrivée remarquable des jeunes dans la vente de médicaments de la rue due à la restriction du marché de l'emploi. L'installation durable de ces jeunes dans cette activité est problématique et incertaine. D’après notre enquête, il ressort que pour les jeunes la vente de médicaments de la rue est une activité transitoire permettant l'épargne nécessaire au financement d'autre métier tel que le transport ou d'un voyage à l'étranger.

Quant aux nationalités, elles sont diverses. On rencontre tout aussi bien des ivoiriens que des étrangers dans le commerce illicite de médicaments de la rue. Nous pouvons affirmer que les activités de vente de médicaments de la rue sont en majorité tenues par les Ivoiriens, 77\%, contre $20 \%$ d'étrangers et $3 \%$ de nationalités non défini.

La prédominance des Ivoiriens s'explique par les difficultés d'emploi que connaît le pays depuis les années 1980 suite à la conjoncture socioéconomique et politique (Institut Nationale de la Statistique, 2005). Aussi la déscolarisation, phénomène récurent qui découle du manque de moyens, a amené beaucoup d'Ivoiriens à s'orienter vers ce secteur informel. En effet, c'est un secteur qui ne demande ni qualification professionnelle ni grands moyens financiers et qui relève de l'initiative personnelle. Parmi les ivoiriens ce sont les mandés qui dominent l'activité. Ils ont le quasi monopole de celle-ci. Ils représentent $75 \%$ des vendeurs ivoiriens et $58 \%$ du 
total des vendeurs; après viennent les Voltaïques qui représentent 12\% des vendeurs ivoiriens et $09 \%$ du total des vendeurs ; les Akan qui représentent $08 \%$ des vendeurs ivoiriens et $06 \%$ du total des vendeurs et enfin les Krou qui représentent 05\% des vendeurs ivoiriens et 04\% du total des vendeurs.

Le commerce constitue l'activité principale des Mandés et des Voltaïques (Sangaré, 2008). En effet, selon Sangaré ce sont les Mandés et les Voltaïques qui ont développé l'activité commerciale en Afrique de l'Ouest. Ils étaient à la croisée des grands axes routiers appelés routes commerciales de l'Afrique de l'Ouest, partant de la Côte d'Ivoire au Ghana en passant par le Mali. Ils développèrent des échanges entre plusieurs produits : la vente de kola (wóro), de tissus, de sel, de poisson séché, etc. Ce carrefour a permis des échanges économiques et culturels et a créé une certaine unité autour du concept « Dioula » (Konaté, 2016). Selon Sangaré (Sangaré, 2008), C'est l'activité commerciale qui a donné lieu à l'appartenance à ce groupe ethnique que constituent les Dioula .Ils parcourraient toutes les semaines, les marchés des différents villages pour écouler leurs produits. C'est un groupe ethnique qui a pratiqué le commerce depuis ses origines. Elles tiennent presque le monopole de la vente de médicaments de la rue.

Une autre caractéristique est le niveau d'instruction qui est un élément d’identification des vendeurs de médicaments de la rue. Ainsi dans le cadre de notre étude, nous avons retenus quatre niveaux d'instruction : les analphabètes, le niveau primaire, le niveau collège et le niveau supérieur. Les analphabètes représentent $69 \%$ des vendeurs de médicaments de la rue et elles sont en majorités de sexe féminin. Le pourcentage élevé des analphabètes s'explique par le fait que le commerce informel ne demande pas un énorme effort intellectuel. Il suffit uniquement pour le vendeur de savoir s'exprimer et compter de l'argent. La présence des vendeurs de médicaments de la rue de niveau d'instruction primaire (21\%), collège/lycée (08\%) et supérieur (02\%) s’explique par la déscolarisation précoce suite à l'abandon ou au manque de moyens financiers permettant de poursuivre les études. Cette déscolarisation oriente les jeunes vers d'autres domaines comme la vente illicite de médicaments dont l'apprentissage et l'exercice ne nécessite ni déploiement de grand moyen financier ni qualification spéciale. Au-delà des niveaux d’instructions cités plus haut, nous avons des personnes ayant terminé les études et qui pratiquent ces activités en raison du chômage croissant en Côte d'Ivoire.

Les médicaments de la rue vendus sur le marché Roxy sont de plusieurs types et d’origines diverses. 


\section{Etat des lieux sur les types de médicaments vendus sur le marché Roxy d'Adjamé}

Deux catégories de médicaments sont à distinguer sur le marché Roxy : la première catégorie est constituée des médicaments disposant d'une autorisation de mise sur le marché et la seconde catégorie est constituée des médicaments de la rue ne disposant pas d’autorisation de mise sur le marché.

En effet, les médicaments de la rue qui possèdent une autorisation de mise sur le marché sont des médicaments que l’on retrouve dans les pharmacies privée ou publique. L'approvisionnement du marché Roxy en ce type de médicaments se fait par les structures de distribution de médicaments authentiques publiques et privées (les grossistes-répartiteurs privés, les unités de fabrication locale, les délégués médicaux, les officines privées et les dons), la Nouvelle Pharmacie de la Santé Publique, les structures de santé. La principale source locale d'approvisionnement de ce type de médicaments de la rue se trouve à Abidjan.

Quant aux médicaments de la rue ne disposant pas d'autorisation de mise sur le marché, le marché Roxy est livré depuis l’étranger. Les plus grandes importations de médicaments viennent du Ghana, de la Guinée, de la Chine, de l'Inde et du Nigeria (Achi, 2003). Ce circuit d'approvisionnement est très complexe, allant du fournisseur étranger aux marchés ivoiriens. Une fois sur le marché illicite, le prix de vente des médicaments est déterminé par le vendeur.

\section{Etat sur les différents procédés de fixation des prix des médicaments de la rue.}

La fixation des prix de médicaments de la rue tient compte du fait que le médicament dispose ou non d'une autorisation de mise sur le marché (A.M.M) et de la situation du médicament dans les pharmacies privées.

Pour les médicaments de la rue provenant aussi bien des unités légale de distribution de médicaments que des pharmacies légales, les prix des médicaments sur le marché illicite sont référencés par le prix du même médicament vendu en pharmacie. Les pharmacies privées représentent un concurrent pour les vendeurs de médicaments de la rue.

Le tableau 1 présente la comparaison du prix de quelques médicaments en pharmacie privée et sur le marché Roxy. D’après le tableau 1, les médicaments sont en général moins chers et plus disponibles sur le marché illicite qu'en pharmacie privée. Ce même constat a été rapporté par Loukouva (Loukouva, 1999) et Angbo-Effi et al (Angbo-Effi et al, 2011) dont l'étude relevait que les médicaments de la rue sont achetés principalement pour leur faible coût. 


\begin{tabular}{|c|c|c|}
\hline Désignation & $\begin{array}{c}\text { Prix en officine } \\
\text { (Franc CFA) }\end{array}$ & $\begin{array}{c}\text { Prix sur le marché } \\
\text { illicite } \\
\text { (Franc CFA) }\end{array}$ \\
\hline RIABAL CP 30MG BT20 & 2130 & $1700-2000$ \\
ZENTEL CPR 400MG BT1 & 1810 & $1500-1750$ \\
LASILIX CPR 4OMG BT30 & 2630 & $1900-2350$ \\
AMPIPHAR GELU 500MG BT 12 & 1130 & $750-975$ \\
DONORMYL CPR SECABLE BT 10 & 2075 & $1600-1850$ \\
RESPIBIEN SOL NASAL FL 15 ML & 1575 & $1000-1250$ \\
DONORMYL CPR SECABLE BT10 & 2075 & $1100-1800$ \\
SELENIUM ACE RICHELET CPR BT24 & 6545 & $4500-5450$ \\
TENSIONORME CPR BT24 & 1535 & $975-1260$ \\
PEPSANE GELEE ORALE 30 SACH10G & 4300 & $2900-3850$ \\
DEXERYL CREM TB 50MG & 2625 & $1975-2600$ \\
DERMOVAL CREM TB10G & 1460 & $800-1350$ \\
LEVOBACT CPR 500MG BT5 & 8030 & $6850-7900$ \\
LIORESAL CPR SECA 10MG BT50 & 7150 & $5200-7000$ \\
DAFLON CPR 500MG BT30 & 4685 & $2500-4500$ \\
FELDENE AMP INJ 20MG BT2 & 1970 & $600-1800$ \\
PONSTYL GELU BT20 & 3050 & $2800-3000$ \\
LISTERINE BAIN BOUCHE 250 ML & 4420 & $2800-4200$ \\
KALEORID LEO LP DRG 600MG B30 & 1340 & $775-1300$ \\
PLAQUENIL CPR 200MG BT30 & 4564 & $3000-4300$ \\
\hline
\end{tabular}

comparaisons de prix de quelques médicaments en pharmacie privée et sur le marché Roxy

Quelques médicaments de la rue font exception cette règle. D’abord, la formation des prix des médicaments de la rue disposant d'une autorisation de mise sur le marché tels que les sirops, présente une situation contraire à la formation des prix des médicaments de la rue décrite précédemment. Le prix d'achat des médicaments sur le marché Roxy est plus élevé que celles vendus dans les pharmacies privées (tableau 2). Le tableau 2 montre la différence de prix entre les médicaments sous la forme de sirops vendus dans les pharmacies privées et sur le marché Roxy. Cette situation est due au fait que, les détaillants du marché Roxy s'approvisionnent en sirops dans les pharmacies privées au prix affiché. En ajoutant leur marge de bénéfice, le prix du sirop de la rue devient supérieur à celui affiché dans les pharmacies privées.

\begin{tabular}{|c|c|c|}
\hline Désignation & $\begin{array}{c}\text { Prix du médicament en } \\
\text { pharmacie privée (Franc } \\
\text { CFA) }\end{array}$ & $\begin{array}{c}\text { Prix du médicaments } \\
\text { au marché Roxy } \\
\text { (Franc CFA) }\end{array}$ \\
\hline APDYL H FLACON 100ML & 2090 & $2600-3100$ \\
AERIUS 60ML & 4160 & $4500-4800$ \\
SEKISAN SOL BUV FL120ML & 2785 & $3000-3500$ \\
BIOZOLE SUSP BUV FL100ML & 1345 & $1400-1850$ \\
MAXILASE 100ML & 2420 & $2600-2950$ \\
FUNGIZONE SUSP BUV NOUR 40ML & 4926 & $5520-5500$ \\
FERCEFOL SP FL150ML & 1605 & $1700-2100$ \\
VIFEX SP FL 100ML & 1495 & $1900-2200$ \\
RANFERON SP FL200ML & 3200 & $3500-3850$ \\
\hline
\end{tabular}

Comparaisons de prix de médicaments sous la forme de sirops en pharmacie privée et sur le marché illicite 
Ensuite, les médicaments rares dont la délivrance par certaines pharmacies privées demande un délai d'attente d'un ou de plusieurs jours aux consommateurs et dont les prix sont élevés, coûtent plus cher sur le marché illicite qu'en pharmacie privée.

Par exemple, des médicaments tels que le Zoladex prescrit pour le traitement du cancer de la prostate métastasé n'est disponible dans la majorité des pharmacies qu'après une préalable commande. Il coûte en officine privée 80000 Francs CFA tandis que sur marché Roxy, il est disponible immédiatement et son prix varie entre 81000 Francs CFA et 85 000 Francs CFA Un autre exemple concerne le médicament Proxeed Plus. Ce médicament est un complément alimentaire spécialement conçu pour optimiser la qualité du sperme et le protéger contre les radicaux libres. Les ingrédients contenus dans Proxeed Plus permettent d'optimiser la santé du sperme en augmentant le nombre, la concentration, la mobilité et la vitesse des spermatozoïdes. En officine, le prix de ce remède, disponible après une commande préalable est de 46000 Francs CFA tandis que sur le marché Roxy il est disponible dans l'immédiat et son prix varie entre 48000 Francs CFA et 50000 Francs CFA. Le dernier exemple concerne le médicament Casodex. Il est indiqué chez les patients atteints d'un cancer de la prostate localement avancé, à haut risque de progression de la maladie. Disponible sous la forme de comprimés, c'est un anti-androgène non stéroïdien spécifique des récepteurs androgéniques, dépourvu de toute autre activité endocrinienne. Il induit une régression du cancer prostatique en bloquant, au niveau des récepteurs, l'activité des androgènes. Disponible après une commande en officine, son prix est de 60000 Francs CFA. Sur le marché illicite, le prix varie entre 62000 Francs CFA et 65000 Francs CFA.

Enfin, dans certains, cas le marché Roxy arrive à se fournir en de nouveaux médicaments avant les pharmacies privées. La fixation des prix de ces médicaments dépend alors de leur(s) principe(s) actif(s).

Par exemple : si le nouveau médicament est à base de quinine, son prix sur le marché illicite sera fonction du prix du médicament à base de quinine le plus élevé disponible sur ce même marché illicite. Le prix du médicament sera revu à la baisse une fois que celui-ci sera disponible dans les pharmacies privées.

La formation du prix des médicaments de la rue disposant d'une autorisation de mise sur le marché est largement influencée par les ruptures de stocks des médicaments en pharmacies. En cas de pénurie d'un médicament dans les officines, les vendeurs du marché Roxy profitent de la rareté de ce médicament pour augmenter son prix sur le marché. Le prix du médicament sur le marché illicite peut alors doubler par rapport à son prix dans les pharmacies privées. 
L’atout principal du marché illicite de médicaments de la rue, selon les clients, est la vente à l'unité. Les médicaments de la rue qui sont vendus à l'unité sont les médicaments qui se présentent sous les formes de comprimés, de gélules, de sachet buvables, d'ampoules, de suppositoires, de tubes.

En pharmacie, tous les médicaments sous les formes galéniques citées plus haut ne sont pas vendus à l'unité. Cela dépend fortement du traitement.

Pour les médicaments qui sont vendus à l'unité, autant dans les pharmacies privées que sur le marché Roxy, les prix pratiqués à Roxy sont nettement inférieurs à ceux pratiqués en pharmacies privées. Pour les médicaments qui sont vendus à l'unité sur le marché illicite, le prix de revient de la boîte de médicament vendu à l'unité est supérieur au prix de vente de la boite en pharmacie privée. A titre d'exemple, une boite de Clamoxyl 500 mg par exemple, qui est un médicament qui permet de traiter plusieurs infections d'origine bactérienne causées par des germes sensibles, contient 12 gélules. Il est vendu au marché Roxy entre 1300 Francs CFA et 1500 Francs CFA (tandis qu'il est vendu dans les pharmacies privées à 1600 Francs CFA). Le prix à l'unité de la gélule au marché Roxy est de 150 Francs CFA. Le prix de revient de la boite est alors de 1800 Francs CFA sur le marché illicite. La boite d'Efferalgan Codéine (paracétamol 500MG et codéine 30MG), qui est utilisé dans le traitement de la douleur, contient 16 comprimés et est vendu sur le marché illicite entre 1600 Francs CFA et 1850 Francs CFA (tandis qu'il est vendu en officine à 2000 Francs CFA). Le prix du comprimé à l'unité sur le marché illicite varie entre 150 Francs CFA et 200 Francs CFA. Le prix de revient de la boite de comprimés vendu à l'unité varie entre 2400 Francs CFA et 3200 Francs CFA.

Concernant les médicaments de la rue ne possédant pas une autorisation de mise sur le marché (A.M.M) vendus sur le marché illicite, ils ont une correspondance dans la catégorie de médicaments de la rue possédant une Autorisation de Mise sur le Marché. La correspondance est établie soit par le nom, soit par le principe actif, soit par la maladie qu'il traite. Pour des médicaments ayant le même nom ou le même principe actif et de même forme galénique, celui possédant une autorisation de mise sur le marché a un prix plus élevé que celui ne possédant pas d'autorisation de mise sur le marché. A titre d'exemple, le médicament Clamoxyl 500MG, est vendu en officine à 1600 Francs CFA. Sur le marché illicite il coûte entre 1300 Francs CFA et 1500 Francs CFA. Le même médicament (Clamoxyl) ne possédant pas d'Autorisation de Mise sur le Marché est vendu entre 850 Francs CFA et 1100 Francs CFA.

Le prix à l'unité des médicaments de la rue ne possédant pas d'autorisation de mise sur le marché est plus abordable que le prix à l'unité des médicaments de la rue possédant une Autorisation de Mise sur le 
Marché. L’exemple suivant va l’illustrer. Il s’agit du médicament Zentel qui est un vermifuge qui appartient à la famille des imidazoles et qui est actif sur un grand nombre de vers parasites de l'intestin. Le comprimé du médicament Zentel possédant une Autorisation de Mise sur le Marché coûte entre 1500 Francs CFA et 1750 Francs CFA, alors que sur le même marché illicite, le prix du comprimé du médicament Zentel ne possédant pas d’autorisation de mise sur le marché varie entre 500 Francs CFA et 1000 Francs CFA.

De façon générale, hormis les sirops, les médicaments rares dont la délivrance par les pharmacies privées demande un délai d'attente d'un ou de plusieurs jours aux consommateurs et des médicaments en rupture de stock dans les pharmacies privées, les prix des médicaments sur le marché illicite sont nettement inférieurs à ceux pratiqués en officine.

\section{Conclusion}

La vente de médicaments de rue se pratique aux yeux de tous. "Aujourd'hui elle donne des vertiges par son ampleur. Elle devient plus dangereuse, plus sournoise et plus professionnelle aussi " (Claire, 2009). Les vendeurs de médicaments de la rue sont des sujets adultes à prédominance de sexe féminin. Tenue à majorité par les ivoiriens, le commerce de vente de médicaments de la rue est le monopole des Mandés. A majorité analphabètes, les vendeurs de médicaments de la rue adoptent des stratégies de fixation de prix afin de vendre, pour être rentable et assurer la pérennité de l'activité.

La fixation des prix des médicaments de la rue au marché Roxy, comme résumé par la figure 2 , dépend de l'origine de l'approvisionnement et de la situation du médicament dans les pharmacies privées. 


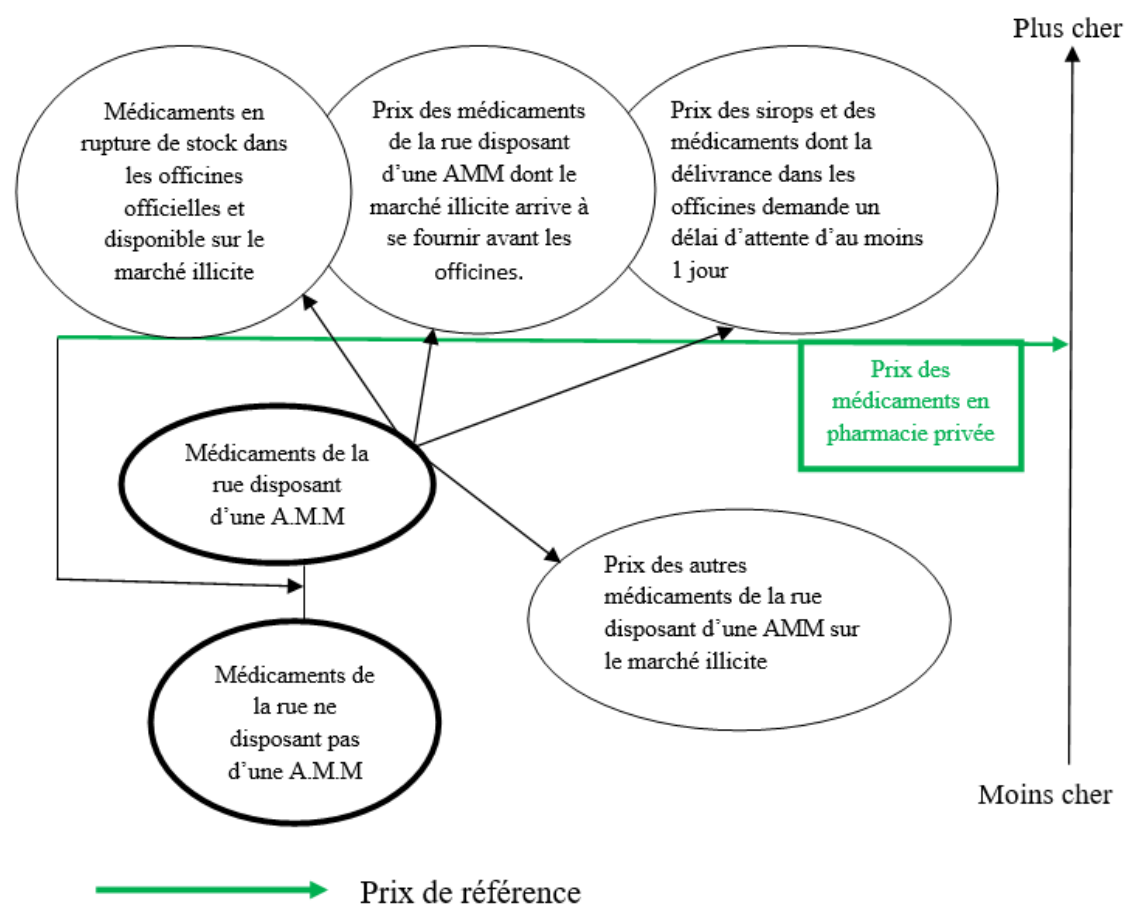

Figure2: Résumé du mécanisme de formation des prix des médicaments de la rue

Les médicaments disposant d'une Autorisation de Mise sur le Marché sont moins cher sur le marché illicite que les médicaments ne disposant pas d'Autorisation de Mise sur le Marché. Elle se positionne par rapport à celles de son concurrent : les pharmacies privées.

Pour les médicaments de la rue disposant d'une Autorisation de Mise sur le Marché tels que : les sirops, les médicaments dont la délivrance dans les pharmacies demande un délai d'attente d'au moins 1 jour, les médicaments dont le marché illicite arrive à se fournir avant les officines et les médicaments en rupture de stock dans les officines officielles sont plus chers sur le marché illicite qu'en officines officielle. A cela il faut ajouter le prix de revient des médicaments vendu à l'unité sur le marché illicite qui est supérieur au prix du médicament en boîte dans les pharmacies.

L’idée selon laquelle les médicaments sur le marché illicite ont un coût moins élève que dans les officines officielles serait à nuancer. Les pouvoirs publics doivent donc sensibiliser la population qui se tourne en majorité vers le marché illicite pour " le coût moins élevé des médicaments ». Certaines pratiques telles que les ventes à l'unité de certains médicaments doivent être encouragé dans les officines officielles et la création d'une assurance maladie pour tous peuvent favoriser l'accessibilité aux médicaments 


\section{References:}

1. Abdallah H. (2000). "La pharmacie par terre» au Niger: une alternative à l'échec de la politique pharmaceutique nationale? Etude de cas à la commune Niamey II. Mémoire de Maîtrise, Université de Ouagadougou, 112 pages.

2. Achi O. (2003). La vente illicite des médicaments en Côte d'Ivoire : contribution à l'évolution d'une politique pharmaceutique. Thèse de doctorat en Droit, université Paris 8, 397 pages.

3. Angbo-Effi O, Kouassi Damus P, Yao Gnissan H, Douba A, Secki R, Kadjo A. (2011). Facteurs déterminant la consommation des médicaments de la rue en milieu urbain. Santé Publique, Vol. 23, p. 455-464.

4. Claire D. (2009). La contrefaction de médicament et son impact sur le continent africain. Thèse pour le diplôme d'Etat de docteur en pharmacie, université de Nantes, 126 pages.

5. Institut National de la Statistique. (2005). Le secteur informel dans l'agglomération d'Abidjan : performance, insertion, perspectives, enquêtes 1,2,3, premier résultat de la phase 2. Côte d'Ivoire, I.N.S, 48p.

6. Koffi M. (2008). L'alphabétisation en Côte d'Ivoire: langues, méthodes et propositions d'aménagement linguistique au regard de la configuration sociolinguistique de la ville d'Abidjan. Thèse de Doctorat unique, Université Abidjan-Cocody, Abidjan.

7. Konate Y. (2016). Le dioula véhiculaire : Situation sociolinguistique en Côte d'Ivoire, Corela, vol.14, $\mathrm{n}^{\circ} 1$.

8. Kouakou Ke. (2007). Description du profil des acheteurs des médicaments de la rue au marché Roxy d'Adjamé. Mémoire de santé publique. INFAS, $74 \mathrm{p}$.

9. Kouamé A. (2007). Commerce de rue et dégradation de l'environnement à Yopougon. Cas des quartiers SIDECI - SICOGI location vente LEM et SOGEPHIA Kouté municipal. Mémoire de Maitrise, université de Cocody-Abidjan, 97P.

10. Loukouva-Chorliet S. (1999). Enquête sur le comportement des acheteurs et des vendeurs de médicaments dans les circuits illicites au Burkina-Faso. Intervention à la table-ronde de remed, le jeudi 14 octobre 1999.

11. Sangare I.(2008). Approche ethnolinguistique des formules de salutation chez les Dioula de Darsalamy au Burkina Faso. Diplôme d'études approfondies option littérature orale, Université de Ouagadougou Burkina Faso, 373P 